\title{
A Triangulation of $\mathbb{C} P^{3}$ as Symmetric Cube of $S^{2}$
}

\author{
Bhaskar Bagchi • Basudeb Datta
}

Received: 25 February 2011 / Revised: 26 April 2012 / Accepted: 25 June 2012 /

Published online: 30 June 2012

(C) Springer Science+Business Media, LLC 2012

\begin{abstract}
The symmetric group $\operatorname{Sym}(d)$ acts on the Cartesian product $\left(S^{2}\right)^{d}$ by coordinate permutation, and the quotient space $\left(S^{2}\right)^{d} / \operatorname{Sym}(d)$ is homeomorphic to the complex projective space $\mathbb{C} P^{d}$. We used the case $d=2$ of this fact to construct a 10 -vertex triangulation of $\mathbb{C} P^{2}$ earlier. In this paper, we have constructed a 124 vertex simplicial subdivision $\left(S^{2}\right)_{124}^{3}$ of the 64-vertex standard cellulation $\left(S_{4}^{2}\right)^{3}$ of $\left(S^{2}\right)^{3}$, such that the $\operatorname{Sym}(3)$-action on this cellulation naturally extends to an action on $\left(S^{2}\right)_{124}^{3}$. Further, the $\operatorname{Sym}(3)$-action on $\left(S^{2}\right)_{124}^{3}$ is "good", so that the quotient simplicial complex $\left(S^{2}\right)_{124}^{3} / \operatorname{Sym}(3)$ is a 30 -vertex triangulation $\mathbb{C} P_{30}^{3}$ of $\mathbb{C} P^{3}$. In other words, we have constructed a simplicial realization $\left(S^{2}\right)_{124}^{3} \rightarrow \mathbb{C} P_{30}^{3}$ of the branched covering $\left(S^{2}\right)^{3} \rightarrow \mathbb{C} P^{3}$.
\end{abstract}

Keywords Triangulated manifolds $\cdot$ Complex projective space $\cdot$ Symmetric power . Product of 2-spheres

\section{Introduction}

Consider the natural action of the symmetric group $\operatorname{Sym}(d)$ of degree $d$ by coordinate permutation on $\left(S^{2}\right)^{d}$, the $d$ th Cartesian power of the 2-sphere. Notice that this action is not free. Indeed, its fixed point set is the diagonally embedded 2-sphere. Nonetheless, it is well known (at least to algebraic geometers) that a miracle happens: the quotient space $\left(S^{2}\right)^{d} / \operatorname{Sym}(d)$ is a manifold! Indeed, the quotient is the $d$-dimensional complex projective space $\mathbb{C} P^{d}$ (see Lemma 2.3 ).

\footnotetext{
B. Bagchi

Theoretical Statistics and Mathematics Unit, Indian Statistical Institute, Bangalore 560 059, India e-mail: bbagchi@isibang.ac.in
}

B. Datta $(\bowtie)$

Department of Mathematics, Indian Institute of Science, Bangalore 560 012, India

e-mail: dattab@math.iisc.ernet.in 
Now, there is the long standing problem of constructing minimal triangulations of $\mathbb{C} P^{d}$, i.e., triangulations using as few vertices as possible. In [1], Arnoux and Marin proved that any triangulation of $\mathbb{C} P^{d}$ requires at least $(d+1)^{2}$ vertices, and equality can hold here only for $d \leq 2$. Of course, $\mathbb{C} P^{1} \cong S^{2}$ has a unique four-vertex triangulation $S_{4}^{2}$, the boundary complex of the three-dimensional simplex. Also, it has long been known that $\mathbb{C} P^{2}$ has a unique 9-vertex triangulation $\mathbb{C} P_{9}^{2}$, first found by Kühnel (see $[1,2,8])$. By the Arnoux-Marin result, for $d \geq 3$, any triangulation of $\mathbb{C} P^{d}$ should require at least $(d+1)^{2}+1$ vertices. But the problem of constructing triangulations attaining this bound remains open. Indeed, for $d \geq 4$, no explicit triangulation of $\mathbb{C} P^{d}$ appears to be known. A representation of $\mathbb{C} P^{d}$ - as a quotient of a torus by $\operatorname{Sym}(d+1)$-was used in Sect. 14 of [1] to obtain a $(d+1)^{2}$-vertex weak cellulation of $\mathbb{C} P^{d}$. The authors of [1] observed that when $d \geq 3$, this weak cellulation cannot be triangulated without adding extra vertices. In [10], Sergeraert uses the Kenzo program to construct simplicial sets (a generalization of simplicial complexes) of complex dimension $d \leq 6$ which "triangulate" topological spaces homotopy equivalent to $\mathbb{C} P^{d}$. It is not known if these are actually triangulations of $\mathbb{C} P^{d}$.

In [3] as well as in this paper, we explore the idea of obtaining reasonably small triangulations of $\mathbb{C} P^{d}$ by triangulating the branched covering $\left(S^{2}\right)^{d} \rightarrow \mathbb{C} P^{d}$ mentioned in the first paragraph. Since the four-vertex triangulation $S_{4}^{2}$ of $S^{2}$ is the boundary complex of the (regular) 3 -simplex $\Delta^{3}$, it follows that a natural $4^{d}$-vertex cellulation of $\left(S^{2}\right)^{d}$, denoted by $\left(S_{4}^{2}\right)^{d}$, sits inside the boundary complex of the $(3 d)$ dimensional polytope $\left(\Delta^{3}\right)^{d}=\Delta^{3} \times \Delta^{3} \times \cdots \times \Delta^{3}$ ( $d$ factors). This polytope is not simplicial; indeed, its facets are simplicial prisms (Cartesian products of simplices). Nevertheless, it is interesting to note that the group $\operatorname{Sym}(d)$ acts on this polytope as a group of isometries via coordinate permutations: mimicking the action of $\operatorname{Sym}(d)$ on $\left(S^{2}\right)^{d}$ mentioned above. Indeed, there is also an isometric diagonal action of Sym(4) on this polytope (inherited from the regular 3-simplex $\Delta^{3}$ ) which commutes with this $\operatorname{Sym}(d)$-action, so we have the group $\operatorname{Sym}(d) \times \operatorname{Sym}(4)$ acting on the polytope $\left(\Delta^{3}\right)^{d}$. The cellulation of $\left(S^{2}\right)^{d}$ (whose facets are the products of $d$ triangles) is stable under this group. In this paper, as in [3], we find it convenient to concentrate on the subgroup $\operatorname{Sym}(d) \times \operatorname{Alt}(4)$. The basic idea is to construct a simplicial subdivision of the polytope, stable under $\operatorname{Sym}(d) \times \operatorname{Alt}(4)$, naturally containing a triangulation of $\left(S^{2}\right)^{d}$, with the same group. The hope is that we may then mod out the group $\operatorname{Sym}(d)$ to obtain a triangulation of $\mathbb{C} P^{d}$ with an automorphism group Alt(4) (since this $\operatorname{Alt}(4)$ commutes with $\operatorname{Sym}(d))$.

But, there is an important question here that needs to be addressed. Let $X$ be a simplicial complex with an automorphism group $G$ and geometric realization $|X|$. Thus, $X$ is a triangulation of the topological space $|X|$. The action of $G$ on $X$ naturally extends to an action on $|X|$ as a group of (piecewise linear) homeomorphisms. We have the quotient topological space $|X| / G$ as well as the quotient simplicial complex $X / G$ (whose vertices correspond to the $G$-orbits on the vertex set of $X$ ). But, is it true that the simplicial complex $X / G$ triangulates the topological space $|X| / G$ ? In general, the answer is a resounding "no". In this paper, we introduce the notion of purity (Definition 2.4) of a group of simplicial automorphisms, and prove (Lemma 2.5) that the conclusion $|X / G| \cong|X| / G$ is valid if and only if the $G$-action on $X$ is pure. The 
notion of purity is technically very demanding, and hard to verify. So, we also introduce the notion of goodness (Definition 2.6) of a group of simplicial automorphisms and prove (Corollary 2.7) that all good actions are pure. This has the advantage that (unlike the notion of purity) goodness of a simplicial automorphism group $G$ of $X$ depends only on the action of $G$ on the 1 -skeleton of $X$. So, goodness is easy to verify.

Thus, if our program for constructing a triangulation of $\mathbb{C} P^{d}$ is to succeed, then the triangulation of $\left(\Delta^{3}\right)^{d}$ must be such that the action of $\operatorname{Sym}(d)$ on this triangulation is good. This will ensure (in view of Lemma 2.3 and Corollary 2.7 ) that the quotient by $\operatorname{Sym}(d)$ of the subcomplex triangulating $\left(S^{2}\right)^{d}$ is indeed a triangulation of $\mathbb{C} P^{d}$.

In [3], we implemented this program for $d=2$. It was shown that the natural 16-vertex cellulation $S_{4}^{2} \times S_{4}^{2}$ of $S^{2} \times S^{2}$ has a natural (and, in some sense unique) 16 -vertex $\operatorname{Sym}(2) \times \operatorname{Alt}(4)$-invariant triangulation $\left(S^{2} \times S^{2}\right)_{16}$ with a good $\operatorname{Sym}(2)$ action, hence obtaining a 10-vertex triangulation of $\mathbb{C} P^{2}$ as a quotient. Thus, there is a triangulation $\left(S^{2} \times S^{2}\right)_{16} \rightarrow \mathbb{C} P_{10}^{2}$ of the branched covering $S^{2} \times S^{2} \rightarrow \mathbb{C} P^{2}$.

In this paper, we consider the case $d=3$. We find that (unlike the case $d=2$ ) the natural 64-vertex cellulation $S_{4}^{2} \times S_{4}^{2} \times S_{4}^{2}$ of $S^{2} \times S^{2} \times S^{2}$ with isometry group $\operatorname{Sym}(3) \times \operatorname{Alt}(4)$ cannot be triangulated in this manner (i.e., retaining the $(\operatorname{Sym}(3) \times$ Alt(4))-action in such a manner that the $\operatorname{Sym}(3)$-action on the resulting complex is good) without adding extra vertices. We obtain a 124-vertex triangulation of $S^{2} \times$ $S^{2} \times S^{2}$ with a 30 -vertex quotient triangulating $\mathbb{C} P^{3}$. Goodness serves as a guiding principle in this construction. Here we prove:

Theorem 1.1 There is a simplicial subdivision $\left(S^{2} \times S^{2} \times S^{2}\right)_{124}$ of the natural 64-vertex cellulation of $S^{2} \times S^{2} \times S^{2}$, which is invariant under the action of $\operatorname{Sym}(3) \times \operatorname{Alt}(4)$. The action of $\operatorname{Sym}(3)$ on this triangulation is good, so that the quotient $\mathbb{C} P_{30}^{3}$ of this triangulation by $\operatorname{Sym}(3)$ is a 30 -vertex triangulation of $\mathbb{C} P^{3}$. Thus, we have a triangulation $\left(S^{2} \times S^{2} \times S^{2}\right)_{124} \rightarrow \mathbb{C} P_{30}^{3}$ of the branched covering $S^{2} \times S^{2} \times S^{2} \rightarrow \mathbb{C} P^{3}$. The branch locus of this covering is the union of three copies of $\left(S^{2} \times S^{2}\right)_{16}$, any two of which intersect in the diagonal $S_{4}^{2}$, and each of which is mapped isomorphically onto a copy of $\left(S^{2} \times S^{2}\right)_{16}$ in $\mathbb{C} P_{30}^{3}$.

Of course, the number 30 of the vertices of the resulting triangulation of $\mathbb{C} P^{3}$ is a far cry from the lower bound of 17 given by Arnoux and Marin. We ran the BISTELLAR program of Lutz (see [9]) on the input $\mathbb{C} P_{30}^{3}$ in order to reduce the number of vertices. The end result was an 18 -vertex triangulation $\mathbb{C} P_{18}^{3}$ with face vector $(18,153,783,2110,3021,2177,622)$. It has Kühnel's $\mathbb{C} P_{9}^{2}$ as an induced subcomplex, with a 9-vertex 6-ball as its simplicial complement. This illustrates the topological construction of $\mathbb{C} P^{3}$ as a compactification of the 6-dimensional ball by $\mathbb{C} P^{2}$.

When we wrote the first version [4] of this paper, we were under the impression that we had found the first explicit triangulation of $\mathbb{C} P^{3}$. Since then, Frank Lutz has informed us that, in 2007, he used an analogue for $\mathbb{C} P^{3}$ of the Banchoff-Kühnel equilibrium triangulation of $\mathbb{C} P^{2}$ [5], in order to construct an 18-vertex triangulation of $\mathbb{C} P^{3}$ with the smaller face vector $(18,153,781,2100,3003,2163,618)$. Notice that it has four fewer facets than the triangulation found by us. Lutz told us that he did not publish his result since he could not achieve the bound of 17 . However, we 
suspect that there may not be any 17-vertex triangulation of $\mathbb{C} P^{3}$. Indeed, note that the two 18-vertex triangulations of $\mathbb{C} P^{3}$ mentioned above are both 2-neighbourly (all possible edges occur). This leads us to ask:

Question: Is it true that any 18 -vertex triangulation of $\mathbb{C} P^{3}$ is 2-neighbourly?

If the answer to this question is in the affirmative, then of course there cannot be any 17-vertex $\mathbb{C} P^{3}$. (If there were, then applying a bistellar zero-move to it one would get an 18 -vertex $\mathbb{C} P^{3}$ with a vertex of degree 7.)

This paper is organized as follows. In Sect. 2, we present a general method for triangulating polytopes (Lemma 2.2) which turns out to be very useful in triangulating $\left(\Delta^{3}\right)^{3}$, as well as the results on group action discussed above. In Sect. 3, we present the details of the construction and the proof of our main theorem. The explicit lists of the facets (in the triangulations obtained) are given in Sect. 4. The branch locus of the simplicial covering map $\left(S^{2} \times S^{2} \times S^{2}\right)_{124} \rightarrow \mathbb{C} P_{30}^{3}$ is also discussed in this section. In the last section, we briefly discuss the 18 -vertex triangulation of $\mathbb{C} P^{3}$ found by us.

\section{Preliminaries and Some Basic Results}

\subsection{Polytopal Complexes and Their Subdivisions}

All affine spaces considered here are finite dimensional. For a set $A$ in an affine space, the smallest affine subspace containing $A$ is called the affine span of $A$ and is denoted by $\operatorname{Aff}(A)$. If $S$ is a finite set in an affine space then the convex hull of $S$ (denoted by $\langle S\rangle$ ) is called a polytope. If $P$ is a polytope in an affine space $H$ then a face of $P$ is either $P$ itself or the intersection of $P$ with a hyperplane $E$ of $H$ such that $P$ is contained in one of the two closed half-spaces determined by $E$. If $F$ is a face of $P$ and $F \neq P$ then $F$ is called a proper face of $P$. The 0 - and 1-dimensional faces of a polytope are called the vertices and edges of the polytope, respectively. The dimension of a polytope $P$ is the dimension of its affine span $\operatorname{Aff}(P)$. If the dimension of a polytope $P$ is $d$ then we say that $P$ is a $d$-polytope. The empty set is a polytope of dimension -1 . Clearly, a $d$-polytope has at least $d+1$ vertices. If it has exactly $d+1$ vertices then it is called a (geometric) simplex. If all the proper faces of a polytope $P$ are simplices then $P$ is called a simplicial polytope. For a polytope $P$, $\partial P$ denotes the topological boundary of $P$ and $\stackrel{\circ}{P}$ denotes the relative interior of $P$. So, $\partial P$ is the union of all the proper faces of $P$ and $\stackrel{\circ}{P}=P \backslash \partial P$.

A polytopal complex $K$ is a finite collection of polytopes in an affine space such that (i) if $P \in K$, then all the faces of $P$ are also in $K$, and (ii) the intersection $P \cap Q$ of two polytopes $P, Q \in K$ is a face of both $P$ and $Q$. The dimension of $K$ (denoted by $\operatorname{dim}(K))$ is the largest dimension of a polytope in $K$. If $K$ is a polytopal complex in an affine space $H$ then the space $|K|:=\cup\{P: P \in K\}$ (with subspace topology of $H$ ) is called the geometric carrier of $K$. If all the polytopes in a polytopal complex $K$ are simplices then $K$ is called a (geometric) simplicial complex.

Two affine subspaces $E$ and $F$ in an affine space $H$ are said to be skew if $\operatorname{dim}(E+F)=\operatorname{dim}(E)+\operatorname{dim}(F)+1$. For two polytopes $P$ and $Q$, if $\operatorname{Aff}(P)$ and 
$\operatorname{Aff}(Q)$ are skew then $\langle P \cup Q\rangle$ is a polytope (denoted by $P * Q$ ) of dimension $\operatorname{dim}(P)+\operatorname{dim}(Q)+1$ and is called the join of $P$ and $Q$. Let $K$ and $L$ be two polytopal complexes. Suppose $\operatorname{Aff}(\alpha)$ and $\operatorname{Aff}(\beta)$ are skew for all $\alpha \in K$ and $\beta \in L$. Then $K * L:=\{\alpha * \beta: \alpha \in K, \beta \in L\}$ is a polytopal complex (called the join of $K$ and $L$ ) of $\operatorname{dimension} \operatorname{dim}(K)+\operatorname{dim}(L)+1$. Clearly, the join of two simplicial complexes is a simplicial complex.

If $P$ is a polytope, the collection $\bar{P}$ of all the faces of $P$ is a polytopal complex, called the face complex of $P$; its geometric carrier is $P$ itself. The collection $\partial \bar{P}$ of all the proper faces of $P$ is another polytopal complex, called the boundary complex of $P$; its geometric carrier is $\partial P$. More generally, if $K$ is a $d$-dimensional polytopal complex and $|K|$ is a $d$-ball, then consider the subcomplex $\partial K$ whose facets are the $(d-1)$-polytopes $A$ in $K$ such that $A$ is a face of exactly one $d$-polytope in $K$. Then $|\partial K|=\partial|K|$. The complex $\partial K$ is called the boundary of $K$. If $K_{1}, K_{2}$ are two polytopal complexes, then their product is the polytopal complex $K_{1} \times K_{2}:=$ $\left\{A_{1} \times A_{2}: A_{1} \in K_{1}, A_{2} \in K_{2}\right\}$. Clearly, we have $\left|K_{1} \times K_{2}\right|=\left|K_{1}\right| \times\left|K_{2}\right|$.

A (polytopal) subdivision of a polytope $P$ is a polytopal complex $L$ whose geometric carrier is $P$. A subdivision $K^{\prime}$ of a polytopal complex $K$ is a polytopal complex such that $\left|K^{\prime}\right|=|K|$ and for each $P \in K^{\prime}$ there exists $Q \in K$ such that $P \subseteq Q$. Clearly, if $Q \in K$ then $K^{\prime}[Q]:=\left\{P \in K^{\prime}: P \subseteq Q\right\}$ is a subdivision of $Q$. We say that $K^{\prime}[Q]$ is the subcomplex of $K^{\prime}$ induced on $Q$. If a subdivision $K^{\prime}$ of a polytopal complex $K$ is a geometric simplicial complex then $K^{\prime}$ is called a simplicial subdivision of $K$ (cf. [11, Chap. 5]). If $M$ is a topological space then a cellulation of $M$ is a polytopal complex $K$ such that $|K|$ is homeomorphic to $M$. If, further, $K$ is a simplicial complex then $K$ is said to be a triangulation of $M$. Clearly, any simplicial subdivision of a cellulation of $M$ is a triangulation of $M$.

Definition 2.1 Let $P$ be a polytope and $x$ be a point of $P$ ( $x$ may or may not be a vertex of $P$ ). Let $C$ be the smallest face of $P$ containing $x$. Then the polytopal complex $\operatorname{Ast}_{P}(x)$, consisting of all the faces of $P$ not containing $C$, is called the antistar of $x$ in $P$.

Lemma 2.2 (The Antistar Lemma) Let $P$ be a d-polytope and $x$ be a point in $P$. Then the collection $\tilde{P}:=\left\{x * D: D \in \operatorname{Ast}_{P}(x)\right\}$ is a polytopal subdivision of $P$. Moreover, if $\operatorname{Ast}_{P}(x)^{\prime}$ is a simplicial subdivision of $\operatorname{Ast}_{P}(x)$ then $\{x * \sigma: \sigma \in$ $\left.\operatorname{Ast}_{P}(x)^{\prime}\right\}$ is a simplicial subdivision of $P$.

Proof Clearly, $\tilde{P}$ is a polytopal complex. Since any polytope in $\tilde{P}$ is of the form $x * D \subseteq P$ for some polytope $D$ in $\operatorname{Ast}_{P}(x)$, it follows that $|\tilde{P}| \subseteq P$.

We now prove $P \subseteq|\tilde{P}|$. If $d \leq 2$, the result is obvious. So, assume $d \geq 3$ and the result is true for smaller values of $d$. Let $C$ be the smallest face of $P$ not containing $x$. Let $x \neq y \in P$. If $y \in\left|\operatorname{Ast}_{P}(x)\right|$ then $y \in|\tilde{P}|$. If $y \in \partial P \backslash\left|\operatorname{Ast}_{P}(x)\right|$ then $y \in F$ for some $(d-1)$-face $F$ of $P$ which contains $C$. By induction hypothesis, $y \in|\tilde{F}| \subseteq|\tilde{P}|$. So, assume that $y \in P \backslash \partial P$. Let $R$ be the ray containing $y$ and initial point $x$. Then $R \cap P$ is a line segment of the form $[x, z]$ for some $z \in \partial P$. If $z \in D$ for some proper face $D$ of $P$ containing $x$, then $[x, z] \subseteq D$ and hence $y \in D \subseteq \partial P$; a contradiction. So, $z \in E$ for some $E \in \operatorname{Ast}_{P}(x)$. This implies that $y \in x * E \subseteq|\tilde{P}|$. This completes the proof of the first statement. The second statement is now obvious. 


\subsection{Simplicial Complexes and Their Geometric Realization}

An abstract simplicial complex (or simply a simplicial complex) is a finite collection of finite sets such that every subset of an element is also an element. For $i \geq 0$, an element of size $i+1$ is called a face of dimension $i$ (or an $i$-face) of the complex. By convention, the empty set is a face of dimension -1 . The dimension of a simplicial complex $X$ (denoted by $\operatorname{dim}(X)$ ) is the maximum of the dimensions of its faces. The 0 - and 1-dimensional faces of a complex are also called the vertices and edges of the complex, respectively. The set $V(X)$ of vertices of a simplicial complex $X$ is called the vertex-set of $X$. A maximal face in a simplicial complex is also called a facet of the complex. Since a simplicial complex is uniquely determined by the set of facets, we sometimes identify a simplicial complex with its set of facets.

If $K, L$ are two simplicial complexes, then a simplicial isomorphism from $K$ to $L$ is a bijection $\pi: V(K) \rightarrow V(L)$ such that for $\sigma \subseteq V(K), \sigma$ is a face of $K$ if and only if $\pi(\sigma)$ is a face of $L$. The complexes $K, L$ are called isomorphic when such an isomorphism exists. We identify two simplicial complexes if they are isomorphic.

The face vector of a $d$-dimensional simplicial complex is the vector $\left(f_{0}, f_{1}\right.$, $\left.\ldots, f_{d}\right)$, where $f_{i}$ is the number of $i$-faces in the complex. It is called $k$-neighbourly if $f_{k-1}=\left(\begin{array}{c}f_{0} \\ k\end{array}\right)$, i.e., if all the possible $(k-1)$-faces occur in the complex.

If $K$ is a geometric simplicial complex and $V$ is the set of vertices of $K$, then $\mathcal{K}:=\{A \subseteq V:\langle A\rangle \in K\}$ is a simplicial complex called the abstract scheme of $K$.

If $X$ is a $d$-dimensional simplicial complex, then let us identify the vertex-set $V$ of $X$ with a set $V$ of points in $\mathbb{R}^{2 d+1}$ such that any subset of $V$ of size at most $2 d+2$ is affinely independent. Then the geometric simplicial complex $\mathcal{X}:=\{\langle\sigma\rangle: \sigma \in X\}$ is called a geometric realization of $X$. The geometric carrier $|\mathcal{X}|$ of $\mathcal{X}$ is also called the geometric carrier of $X$ and is denoted by $|X|$. Clearly, $X$ is isomorphic to the abstract scheme of $\mathcal{X}$. If a topological space $M$ is homeomorphic to $|X|$ then we say $X$ triangulates $M$. It is trivial that isomorphic finite simplicial complexes have homeomorphic geometric carriers. If $|X|$ is a topological manifold (respectively, $d$-sphere) then $X$ is called a triangulated manifold (respectively, triangulated $d$-sphere). If $|X|$ is a pl manifold (with the $\mathrm{pl}$ structure induced by $X$ ) then $X$ is called a combinatorial manifold.

For a finite set $V$ with $d+2(d \geq-1)$ elements, the collection $\bar{V}$ of all the subsets of $V$ is a simplicial complex which triangulates the $(d+1)$-ball. The complex $\bar{V}$ is called the standard $(d+1)$-ball and is also denoted by $D_{d+2}^{d+1}(V)$ (or simply by $\left.D_{d+2}^{d+1}\right)$. It is the abstract scheme of the face complex of a $(d+1)$-simplex. The collection $\partial \bar{V}$ of all the proper subsets of $V$ is a simplicial complex (a subcomplex of $\bar{V}$ ) which triangulates the $d$-sphere $S^{d}$. The complex $\partial \bar{V}$ is called the standard $d$-sphere and is also denoted by $S_{d+2}^{d}(V)$ (or simply by $S_{d+2}^{d}$ ). Note that $S_{d+2}^{d}$ is the abstract scheme of the boundary complex of a $(d+1)$-simplex. (Generally, we write $X=X_{n}^{d}$ to indicate that $X$ has $n$ vertices and dimension $d$.)

\subsection{Group Actions and Quotients}

Consider $\left(S^{2}\right)^{d}$, the Cartesian product of $d$ copies of the 2-sphere $S^{2}$. The symmetric group $\operatorname{Sym}(d)$ acts on $\left(S^{2}\right)^{d}$, by coordinate permutations, as a group of homeomorphisms:

$$
\pi:\left(x_{1}, \ldots, x_{d}\right) \mapsto\left(x_{\pi(1)}, \ldots, x_{\pi(d)}\right), \quad \text { for } \pi \in \operatorname{Sym}(d) .
$$


Lemma 2.3 The quotient space $\left(S^{2}\right)^{d} / \operatorname{Sym}(d)$ is homeomorphic to the d-dimensional complex projective space $\mathbb{C} P^{d}$.

Proof We use the usual identification of $S^{2}$ with $\mathbb{C} P^{1}$ ("the Riemann sphere") via stereographic projection. So, we need to show that $\left(\mathbb{C} P^{1}\right)^{d} / \operatorname{Sym}(d) \cong \mathbb{C} P^{d}$. Recall the usual description of $\mathbb{C} P^{d}$ by homogeneous coordinates: $\mathbb{C} P^{d}=\left(\mathbb{C}^{d+1} \backslash\right.$ $\{(0, \ldots, 0)\}) / \sim$, where the binary relation $\sim$ is defined by $\left(y_{0}, \ldots, y_{d}\right) \sim\left(x_{0}, \ldots, x_{d}\right)$ if and only if $y_{i}=\lambda x_{i}, 0 \leq i \leq d$, for some $\lambda \in \mathbb{C} \backslash\{0\}$. As usual, $\left[x_{0}, x_{1}, \ldots, x_{d}\right]$ denotes the $\sim$-class containing $\left(x_{0}, \ldots, x_{d}\right)$. Now consider the map $\varphi:\left(\mathbb{C} P^{1}\right)^{d} \rightarrow \mathbb{C} P^{d}$ defined as

$$
\varphi\left(\left[z_{1}, w_{1}\right], \ldots,\left[z_{d}, w_{d}\right]\right)=\left[\alpha_{0}, \alpha_{1}, \ldots, \alpha_{d}\right],
$$

where the complex numbers $\alpha_{0}, \alpha_{1}, \ldots, \alpha_{d}$ are determined by the identity

$$
\sum_{j=0}^{d} \alpha_{j} X^{j} Y^{d-j}=\prod_{k=1}^{d}\left(z_{k} X+w_{k} Y\right) .
$$

Since multiplication in $\mathbb{C}$ is commutative, $\varphi$ is well-defined. It is clearly continuous. Since the field $\mathbb{C}$ is algebraically closed, each homogeneous polynomial of degree $d(\neq 0)$ in $\mathbb{C}[X, Y]$ has exactly $d$ roots in $\mathbb{C} P^{1}$ (counting with multiplicity). Also, modulo multiplication by non-zero scalars, such a polynomial is uniquely determined by its zeros, and in turn it determines its zero-set except for permutations. Thus, $\varphi$ is onto and its fibres are precisely the $\operatorname{Sym}(d)$-orbits in its domain. Therefore, $\varphi$ induces a continuous bijection $\hat{\varphi}:\left(\mathbb{C} P^{1}\right)^{d} / \operatorname{Sym}(d) \rightarrow \mathbb{C} P^{d}$. Since both domain and range are compact Hausdorff spaces, $\hat{\varphi}$ is a homeomorphism.

One says that $\mathbb{C} P^{d}$ is the $d$ th symmetric power of $S^{2}=\mathbb{C} P^{1}$. In [3], we used the case $d=2$ of this lemma to construct a 10-vertex triangulation of $\mathbb{C} P^{2}$. Here we use its $d=3$ case to obtain an explicit triangulation of $\mathbb{C} P^{3}$.

Let $G$ be a group of simplicial automorphisms of a simplicial complex $X$. The action of $G$ on $X$ is called proper if any two distinct vertices from each $G$-orbit are at distance at least 3 in the 1 -skeleton of $X$ (i.e., $\operatorname{lk}_{X}(x) \cap \operatorname{lk}_{X}(y)=\emptyset$ for $x \neq y$ in any $G$-orbit). If the action of $G$ is proper and $X$ is a triangulated manifold, then the quotient complex is also a triangulated manifold (cf. [6, Proposition 1.1]). Here we define the following.

Definition 2.4 Let $G$ be a group of simplicial automorphisms of a simplicial complex $X$. We shall say that the action of $G$ on $X$ is pure if it satisfies: (a) whenever $u$, $v$ are distinct vertices from the same $G$-orbit, $u v$ is a non-edge of $X$, and (b) for each $G$-orbit $\theta \subseteq V(X)$ and each $\alpha \in X$, the stabilizer $G_{\alpha}$ of $\alpha$ in $G$ acts transitively on $\theta \cap V\left(\operatorname{lk}_{X}(\alpha)\right)$.

The "if" part of the following lemma is from [3]. This lemma clarifies the significance of the notion of purity.

Lemma 2.5 (The Purity Lemma) Let $G$ be a group of simplicial automorphisms of a simplicial complex $X$. Let $q: V(X) \rightarrow V(X) / G$ denote the quotient map, and 
$X / G:=\{q(\alpha): \alpha \in X\}$. Extend the action of $G$ on $V(X)$ to an action of $G$ on $|X|$ piecewise linearly, i.e., affinely on the geometric carrier of each face. Then there is a homeomorphism from $|X| / G$ onto $|X / G|$ induced by $|q|:|X| \rightarrow|X / G|$ if and only if the action of $G$ on $X$ is pure. In consequence, when the $G$-action on $X$ is pure, we have $|X / G|=|X| / G$.

Proof First suppose that the $G$-action is pure. The condition (a) ensures that the quotient map $q$ is one-to-one on each face of $X$. The simplicial map $q: X \rightarrow X / G$ induces a piecewise linear continuous map $|q|$ from $|X|$ onto $|X / G|$.

Claim. The fibres of $q: X \rightarrow X / G$ are precisely the $G$-orbits on simplices of $X$ (that is, if $\alpha, \alpha^{\prime} \in X$ are such that $q(\alpha)=q\left(\alpha^{\prime}\right)$ then there exists $g \in G$ such that $\left.g(\alpha)=\alpha^{\prime}\right)$.

We prove the claim by induction on $k=\operatorname{dim}(\alpha)=\operatorname{dim}\left(\alpha^{\prime}\right)$. The claim is trivial for $k=-1$. So, assume $k \geq 0$ and the claim is true for all smaller dimensions. Choose a face $\beta \subseteq \alpha$ of dimension $k-1$, and let $\beta^{\prime} \subseteq \alpha^{\prime}$ be such that $q\left(\beta^{\prime}\right)=q(\beta)$. By induction hypothesis, $\beta^{\prime}$ and $\beta$ are in the same $G$-orbit. Therefore, applying a suitable element of $G$, we may assume, without loss of generality, that $\beta^{\prime}=\beta$. Let $\alpha=\beta \cup$ $\{x\}, \alpha^{\prime}=\beta \cup\left\{x^{\prime}\right\}$. Then $q(x)=q\left(x^{\prime}\right)$, i.e., $x$ and $x^{\prime}$ are in the same $G$-orbit. Now, by assumption (b), there is a $g \in G_{\beta}$ such that $g(x)=x^{\prime}$. Then $g(\alpha)=\alpha^{\prime}$. This proves the claim.

In the presence of condition (a), the claim ensures that the fibres of $|q|$ are precisely the $G$-orbits on points of $|X|$. Hence $|q|$ induces the required homeomorphism between $|X| / G$ and $|X / G|$.

Now assume that the map between $|X| / G$ and $|X / G|$ induced by $|q|$ is a homeomorphism. Clearly, condition (a) holds since the quotient map preserves dimension. So, (a) holds, and the fibres of $q$ are the $G$-orbits in $X$. Let $\alpha, \theta$ be as in (b), and let $x \neq y$ be vertices in $\theta \cap \operatorname{lk}(\alpha)$. Then $q(\alpha \cup\{x\})=q(\alpha \cup\{y\})$ and hence there is an element $g$ of $G$ such that $\alpha \cup\{y\}=g(\alpha \cup\{x\})$. Since $y \in \theta$, (a) ensures that $\theta$ is disjoint from the vertex-set of $\alpha$, so that we must have $y=g(x)$. Hence $g \in G_{\alpha}$, and condition (b) holds. Thus the $G$-action on $X$ is pure.

Definition 2.6 Let $G$ be a group of simplicial automorphisms of a simplicial complex $X$. We shall say that the group $G$ is good (or, the action of $G$ on $X$ is good) if it satisfies: whenever $x \neq y$ are vertices of $X$ from a common $G$-orbit, then $x y$ is a non-edge, and there is $g \in G$ such that $g(x)=y$ and $g$ fixes all the vertices in $\operatorname{lk}(x) \cap \operatorname{lk}(y)$.

Corollary 2.7 Let $G$ be a group of simplicial automorphisms of a simplicial complex $X$. If $G$ is good then the action of $G$ is pure and hence $|X / G|=|X| / G$.

Proof Clearly $G$ satisfies condition (a). We need to show that $G$ also satisfies condition (b) for purity. So, let $\alpha, \theta$ be as in (b) and $x \neq y$ be vertices in $\theta \cap \operatorname{lk}(\alpha)$. Then the element $g$ of $G$ (given by Definition 2.6) fixes all the vertices of $\alpha$, and hence $g \in G_{\alpha}$. 
Remark 2.8 Goodness of $G$ may not be necessary for the purity of its action. However, goodness has the virtue of simplicity: to verify it, one need only examine the action of $G$ on the 1-skeleton of $X$. Notice also that purity of $G$ implies that the stabilizer of any face fixes the face vertex-wise, so that the definition of a good action is not as stringent as it may appear. Note that if the action of $G$ on $X$ is proper then $G$ is good and hence the quotient complex $X / G$ triangulates $|X| / G$. Moreover, if $X$ is a triangulated manifold then the quotient map $q: X \rightarrow X / G$ is a covering.

In the following, we shall say that an isometry group $G$ of a geometric simplicial complex is good (or pure) if the induced action of $G$ on its abstract scheme is good (respectively, pure).

\section{Proof of Theorem 1.1}

By a slight abuse of notation, we let $S_{4}^{2}$ denote the boundary complex of the regular tetrahedron in $\mathbb{R}^{3}$ whose vertices are $x_{1}=(1,-1,-1), x_{2}=(-1,1,-1)$, $x_{3}=(-1,-1,1)$ and $x_{4}=(1,1,1)$. We use the notation $x_{i j k}$ to denote the vertex $\left(x_{i}, x_{j}, x_{k}\right)$ of $S_{4}^{2} \times S_{4}^{2} \times S_{4}^{2}$ in $\mathbb{R}^{9}$. So, the vertex-set of $S_{4}^{2} \times S_{4}^{2} \times S_{4}^{2}$ is $\left\{x_{i j k}: 1 \leq\right.$ $i, j, k \leq 4\}$. (With our notation, for instance $x_{214}=(-1,1,-1,1,-1,-1,1,1,1)$.) Observe that $\operatorname{Sym}(3)$ acts on these vertices by permuting the positions of the three subscripts and this action induces the same action on $\left|S_{4}^{2}\right| \times\left|S_{4}^{2}\right| \times\left|S_{4}^{2}\right|$ as was used in Lemma 2.3. Also, the alternating group Alt(4) (which is an index-two subgroup of the full isometry group of the regular tetrahedron) has an induced diagonal action on $S_{4}^{2} \times S_{4}^{2} \times S_{4}^{2}$. Clearly, Alt(4) commutes with $\operatorname{Sym}(3)$, so we have the isometry group $\operatorname{Sym}(3) \times \operatorname{Alt}(4)$ at our disposal. Notice that, in view of our notation for the vertices, Alt(4) acts on the vertices by permuting the values of the subscripts.

Under the action of the group $\operatorname{Sym}(3) \times \operatorname{Alt}(4)$, the splitting of the faces of $S_{4}^{2} \times$ $S_{4}^{2} \times S_{4}^{2}$ into orbits is as indicated in Table 1. (The numerical suffix decorating a representative face gives the length of the corresponding orbit.)

Lemma 3.1 There does not exist any 64-vertex $\operatorname{Sym}(3)$-invariant simplicial subdivision of the 3-skeleton $W^{3}$ of the polytopal complex $\left(S_{4}^{2}\right)^{3}$ on which the induced $\operatorname{Sym}(3)$ action is pure.

Proof Since the Sym(3)-action is pure, by condition (a), $x_{l i j} x_{l j i}$ is not an edge for all $l$ and $i \neq j$. So, to subdivide $x_{l} \times x_{i} x_{j} \times x_{i} x_{j}$, we have to take $x_{l i i} x_{l j j}$ an edge. Since $x_{l i j} x_{l i k}$ is an edge (in $W^{3}$ ), by condition (b), $x_{l j i} x_{l i k}$ is a non-edge for $i, j, k$ distinct. Then, from $x_{l} \times x_{i} x_{j} \times x_{i} x_{k}, x_{l i i} x_{l j k}$ is an edge. Similarly, $x_{i l i} x_{j l j}, x_{i i l} x_{j j l}, x_{i l i} x_{j l k}$ $x_{i i l} x_{j k l}$ are edges for all $l, k$ and $i \neq j$.

Consider the 3 -cell $C=x_{1} x_{2} \times x_{1} x_{3} \times x_{2} x_{3}$ in $W^{3}$. Since $x_{133} x_{123}, x_{133} x_{122}$, $x_{112} x_{332}, x_{113} x_{123}, x_{113} x_{223}$ are edges in $X^{3}$, by condition (b) of purity, $x_{133} x_{213}$, $x_{133} x_{212}, x_{112} x_{233}, x_{113} x_{132}, x_{113} x_{232}$ are non-edges in $X^{3}$. Since $x_{113} x_{132}$ is a nonedge, $\alpha:=x_{112} x_{113} x_{133}$ is a triangle of $X^{3}$. Then $X^{3}$ must contain a tetrahedron $\sigma \supseteq \alpha$ with $V(\sigma) \subseteq V(C)$, and there is no valid choice of $\sigma$ because of the abovementioned five non-edges (cf. Fig. 1). 
Table 1 Orbits of cells

Cells List of orbits

Vertices $\quad\left\{\left(x_{1}, x_{1}, x_{1}\right)\right\}_{4},\left\{\left(x_{1}, x_{1}, x_{2}\right)\right\}_{36},\left\{\left(x_{1}, x_{2}, x_{3}\right)\right\}_{24}$

Edges $\quad\left\{x_{1} \times x_{1} \times x_{1} x_{2}\right\}_{36},\left\{x_{1} \times x_{1} \times x_{2} x_{3}\right\}_{36},\left\{x_{1} \times x_{2} \times x_{1} x_{2}\right\}_{36}$,

$\left\{x_{1} \times x_{2} \times x_{1} x_{3}\right\}_{72},\left\{x_{1} \times x_{2} \times x_{1} x_{4}\right\}_{72},\left\{x_{1} \times x_{2} \times x_{3} x_{4}\right\}_{36}$

2-Cells $\quad\left\{x_{1} \times x_{1} \times x_{1} x_{2} x_{3}\right\}_{36},\left\{x_{1} \times x_{1} \times x_{2} x_{3} x_{4}\right\}_{12},\left\{x_{1} \times x_{2} \times x_{1} x_{2} x_{3}\right\}_{72},\left\{x_{1} \times x_{2} \times x_{1} x_{3} x_{4}\right\}_{72}$, $\left\{x_{1} \times x_{1} x_{2} \times x_{1} x_{2}\right\}_{36},\left\{x_{1} \times x_{1} x_{2} \times x_{1} x_{3}\right\}_{72},\left\{x_{1} \times x_{1} x_{2} \times x_{3} x_{4}\right\}_{72},\left\{x_{1} \times x_{1} x_{3} \times x_{3} x_{4}\right\}_{72}$, $\left\{x_{1} \times x_{1} x_{4} \times x_{3} x_{4}\right\}_{72},\left\{x_{1} \times x_{2} x_{3} \times x_{2} x_{3}\right\}_{36},\left\{x_{1} \times x_{2} x_{4} \times x_{3} x_{4}\right\}_{72}$

3-Cells $\quad\left\{x_{h} \times x_{1} x_{2} \times x_{1} x_{2} x_{3}\right\}_{72},\left\{x_{h} \times x_{1} x_{4} \times x_{1} x_{2} x_{3}\right\}_{72}, 1 \leq h \leq 4$,

$\left\{x_{1} x_{2} \times x_{1} x_{2} \times x_{1} x_{2}\right\}_{6},\left\{x_{1} x_{2} \times x_{1} x_{2} \times x_{1} x_{3}\right\}_{36},\left\{x_{1} x_{2} \times x_{1} x_{2} \times x_{1} x_{4}\right\}_{36}$, $\left\{x_{1} x_{2} \times x_{1} x_{2} \times x_{3} x_{4}\right\}_{18},\left\{x_{1} x_{2} \times x_{1} x_{3} \times x_{1} x_{4}\right\}_{24},\left\{x_{1} x_{2} \times x_{1} x_{3} \times x_{2} x_{3}\right\}_{24}$, $\left\{x_{1} x_{2} \times x_{2} x_{3} \times x_{3} x_{4}\right\}_{36},\left\{x_{1} x_{2} \times x_{2} x_{4} \times x_{3} x_{4}\right\}_{36}$

4-Cells $\quad\left\{x_{1} \times x_{1} x_{2} x_{3} \times x_{1} x_{2} x_{3}\right\}_{36},\left\{x_{1} \times x_{1} x_{2} x_{3} \times x_{1} x_{2} x_{4}\right\}_{72},\left\{x_{3} \times x_{1} x_{2} x_{3} \times x_{1} x_{2} x_{4}\right\}_{72}$, $\left\{x_{4} \times x_{1} x_{2} x_{3} \times x_{1} x_{2} x_{3}\right\}_{12},\left\{x_{1} x_{2} \times x_{1} x_{2} \times x_{1} x_{2} x_{3}\right\}_{36},\left\{x_{1} x_{2} \times x_{1} x_{2} \times x_{1} x_{3} x_{4}\right\}_{36}$, $\left\{x_{1} x_{2} \times x_{1} x_{3} \times x_{1} x_{2} x_{3}\right\}_{72},\left\{x_{1} x_{2} \times x_{1} x_{3} \times x_{1} x_{3} x_{4}\right\}_{72},\left\{x_{1} x_{2} \times x_{1} x_{3} \times x_{2} x_{3} x_{4}\right\}_{72}$, $\left\{x_{1} x_{2} \times x_{1} x_{4} \times x_{1} x_{3} x_{4}\right\}_{72},\left\{x_{1} x_{2} \times x_{3} x_{4} \times x_{1} x_{2} x_{3}\right\}_{72}$

5-Cells $\quad\left\{x_{1} x_{2} \times x_{1} x_{3} x_{4} \times x_{2} x_{3} x_{4}\right\}_{36},\left\{x_{1} x_{3} \times x_{1} x_{2} x_{3} \times x_{2} x_{3} x_{4}\right\}_{72},\left\{x_{1} x_{3} \times x_{1} x_{3} x_{4} \times x_{2} x_{3} x_{4}\right\}_{72}$, $\left\{x_{1} x_{3} \times x_{2} x_{3} x_{4} \times x_{2} x_{3} x_{4}\right\}_{36},\left\{x_{3} x_{4} \times x_{1} x_{3} x_{4} \times x_{2} x_{3} x_{4}\right\}_{36},\left\{x_{3} x_{4} \times x_{2} x_{3} x_{4} \times x_{2} x_{3} x_{4}\right\}_{36}$

6-Cells $\quad\left\{x_{1} x_{2} x_{3} \times x_{1} x_{2} x_{3} \times x_{1} x_{2} x_{3}\right\}_{4},\left\{x_{1} x_{2} x_{3} \times x_{1} x_{2} x_{3} \times x_{1} x_{3} x_{4}\right\}_{36}$, $\left\{x_{1} x_{2} x_{3} \times x_{1} x_{2} x_{4} \times x_{1} x_{3} x_{4}\right\}_{24}$

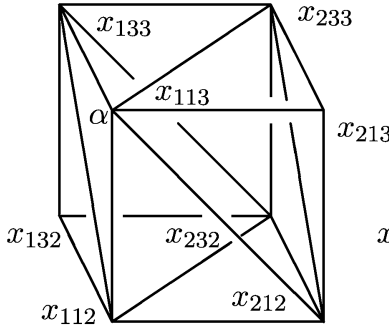

$C=x_{1} x_{2} \times x_{1} x_{3} \times x_{2} x_{3}$

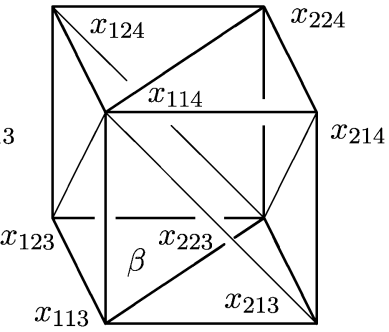

$D=x_{1} x_{2} \times x_{1} x_{2} \times x_{3} x_{4}$ (Case 1)

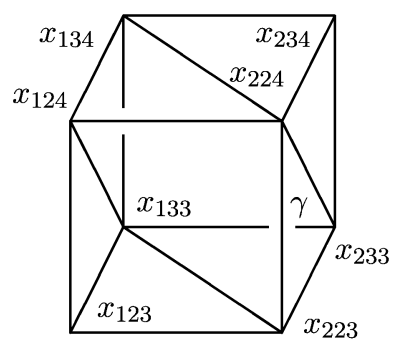

$E=x_{1} x_{2} \times x_{2} x_{3} \times x_{3} x_{4}$

Fig. 1 Subdivisions of 3-cubes

If follows from Lemma 3.1 that any $\operatorname{Sym}(3) \times \operatorname{Alt}(4)$-invariant simplicial subdivision $X$ of $\left(S_{4}^{2}\right)^{3}$ on which $\operatorname{Sym}(3)$-action is pure contains at least $64+24=88$ vertices. Here we prove:

Lemma 3.2 If $X^{3}$ is a $\operatorname{Sym}(3) \times \operatorname{Alt}(4)$-invariant simplicial subdivision of the 3 -skeleton $W^{3}$ polytopal complex $S_{4}^{2} \times S_{4}^{2} \times S_{4}^{2}$ such that $\operatorname{Sym}(3)$-action on $X^{3}$ is pure, then $X^{3}$ has more than 88 vertices.

Proof If possible, let $X^{3}$ be a simplicial subdivision of $W^{3}$ with 88 vertices. Then $X^{3}$ induces simplicial subdivisions on the 3-cells $D=x_{1} x_{2} \times x_{1} x_{2} \times x_{3} x_{4}, E=$ $x_{1} x_{2} \times x_{2} x_{3} \times x_{3} x_{4}$ and $F=x_{1} x_{2} \times x_{2} x_{4} \times x_{3} x_{4}$ without new vertices. 
Consider the square $S=x_{1} \times x_{1} x_{2} \times x_{3} x_{4}$. Clearly, either $x_{123} x_{114} \in X^{3}$ or $x_{124} x_{113} \in X^{3}$.

Case 1: $x_{123} x_{114}$ is an edge. By $\operatorname{Sym}(3) \times \operatorname{Alt}(4)$-action, $x_{213} x_{114}, x_{214} x_{223}, x_{124} x_{223}$ are edges in $X^{3}$. Then only possible tetrahedron containing $\beta=x_{113} x_{123} x_{223}$ in $D$ is $x_{113} x_{123} x_{223} x_{114}$. Then (the long diagonal) $x_{114} x_{223}$ is an edge. Then (by Alt(4)action), $x_{224} x_{331}$ is an edge.

Since $x_{224} x_{331}, x_{223} x_{143}, x_{233} x_{214}, x_{233} x_{213}, x_{223} x_{243}$ are edges in $X^{3}$, by condition (b) of purity, $x_{224} x_{133}, x_{223} x_{134}, x_{233} x_{124}, x_{233} x_{123}, x_{223} x_{234}$ are non-edges in $X^{3}$ and hence $\gamma:=x_{223} x_{224} x_{233} \subseteq E$ is a triangle in $X^{3}$. Then $X^{3}$ must contain a tetrahedron $\sigma \supseteq \gamma$ with $V(\sigma) \subseteq V(D)$, and there is no valid choice of $\sigma$ because of the above-mentioned five non-edges (cf. Fig. 1). This is a contradiction since $X^{3}$ induces a subdivision on $E$.

Case 2: $x_{124} x_{113}$ is an edge. By similar argument as in Case 1, it follows that $x_{113} x_{224} \subseteq D$ is an edge in $X^{3}$. Then (by Alt(4)-action), $x_{441} x_{223}$ is an edge and hence $x_{144} x_{223}$ is a non-edge. Then (as in Case 1) $X^{3}$ cannot induce any simplicial subdivision on $F$; a contradiction.

New Vertices In the light of Lemma 3.1, we insert the barycentre $v_{321}$ of $C=x_{1} x_{2} \times$ $x_{1} x_{3} \times x_{2} x_{3}$. We subdivide $S=x_{1} \times x_{1} x_{2} \times x_{3} x_{4}$ by adding the edge $x_{123} x_{114}$ as in Case 1 in the proof of Lemma 3.2. Then, by Lemma 3.2, we have to add a new vertex in $D$ or in $E$. We add the barycentre $u_{11}$ of $E=x_{1} x_{2} \times x_{2} x_{3} \times x_{3} x_{4}$. In coordinates, we have $v_{321}=(0,0,-1,0,-1,0,-1,0,0)$ and $u_{11}=(0,0,-1,-1,0,0,0,0,1)$.

Notice that the 24 cells in the $\operatorname{Sym}(3) \times \operatorname{Alt}(4)$-orbit of $C$ are $x_{j} x_{k} \times x_{i} x_{k} \times$ $x_{i} x_{j}, i, j, k$ distinct. By the assumed $\operatorname{Sym}(3) \times \operatorname{Alt}(4)$-symmetry, we must choose the barycentres $v_{i j k}$ of these cells as new vertices. Notice that, by our choice of notation, $\operatorname{Sym}(3) \times \operatorname{Alt}(4)$ has its natural action (in terms of the subscripts and their positions) on the vertices $v_{i j k}, i, j, k \in\{1,2,3,4\}$ distinct.

Similarly, the 36 cells in the $\operatorname{Sym}(3) \times \operatorname{Alt}(4)$-orbit of $E$ may be written uniquely as $E_{\alpha, \beta}=(\alpha, \beta)(E),(\alpha, \beta) \in \operatorname{Alt}(3) \times \operatorname{Alt}(4)$. By symmetry, we must choose the barycentre $u_{\alpha, \beta}$ of $E_{\alpha, \beta}$ as a new vertex. For notational convenience, we label the elements of $\operatorname{Sym}(3)$ and $\operatorname{Alt}(4)$ as follows:

$$
\begin{aligned}
\operatorname{Sym}(3)=\{ & \left.\alpha_{1}=\mathrm{id}, \alpha_{2}=(123), \alpha_{3}=(132), \alpha_{4}=(13), \alpha_{5}=(23), \alpha_{6}=(12)\right\}, \\
\operatorname{Alt}(4)=\{ & \beta_{1}=\mathrm{id}, \beta_{2}=(123), \beta_{3}=(124), \beta_{4}=(132), \beta_{5}=(134), \beta_{6}=(12)(34), \\
& \beta_{7}=(14)(23), \beta_{8}=(142), \beta_{9}=(234), \beta_{10}=(143), \\
& \left.\beta_{11}=(243), \beta_{12}=(13)(24)\right\} .
\end{aligned}
$$

We use the simpler notation $u_{i j}$ for the new vertex $u_{\alpha_{i}, \beta_{j}}$. One may check that $u_{i+3, j-6}=u_{i, j}$, for $7 \leq j \leq 12$, where the addition in the first index is modulo 6 . Then the new vertices are $u_{i j}, 1 \leq i, j \leq 6$ and $v_{i j k}, i, j, k \in\{1,2,3,4\}$ distinct.

Remark 3.3 The argument above shows that the required simplicial subdivision of $W$ needs at least $64+24+18=106$ vertices. We now see that it may be possible to obtain a triangulation with 106 vertices. However, this would not reduce the number (thirty) of vertices in the quotient $\mathbb{C} P^{3}$. So, we have decided to retain in this paper 
the original 124-vertex triangulation of $W$ obtained by us, rather than implement the elaborate changes necessary to present an 106-vertex triangulation. Moreover, the 124-vertex triangulation presented here seems to have the more natural branch locus (cf. the discussion in Sect. 4.3).

Now we sketch a proof of Theorem 1.1. Further details may be found in the arXiv [4].

Proof of Theorem 1.1 For $0 \leq k \leq 6$, let $W^{k}$ denote the $k$-skeleton of $W=S_{4}^{2} \times$ $S_{4}^{2} \times S_{4}^{2}$. That is, $W^{k}$ is the subcomplex of $W$ consisting of all its polytopes of dimension at most $k$. For $0 \leq k \leq 6$, we construct a simplicial subdivision $X^{k}$ of $W^{k}$. The construction is by finite induction on $k$, with the following constraints: (i) $X^{0}=W^{0}$, $X^{1}=W^{1}$, (ii) $X^{k-1} \subseteq X^{k}$ for $k \geq 1$, (iii) $X^{k}$ is stable under the isometry group $\operatorname{Sym}(3)$ of $W$, (iv) the $\operatorname{Sym}(3)$-action on $X^{k}$ is good. There are choices for $X^{2}$ subject to these constraints. We choose the most symmetrical of these choices, with an isometry group $\operatorname{Sym}(3) \times \operatorname{Alt}(4)$. To keep the construction under control, we also require that $(\mathrm{v})$ for $k \geq 3, X^{k}$ inherits the isometry group $\operatorname{Sym}(3) \times \operatorname{Alt}(4)$ from $X^{k-1}$. We refuse to subdivide those cells of $W$ which are already simplices. In particular, $X^{0}=W^{0}, X^{1}=W^{1}$. Because of the assumption (v), it suffices to give the subdivisions in $X^{k}, k \geq 2$, of the non-simplicial $k$-cells from Table 1. Because of the $\operatorname{Sym}(3) \times \operatorname{Alt}(4)$-symmetry, the subdivision propagates to the whole of $W^{k}$. Of course, to be consistent, the chosen subdivision $\hat{\alpha}$ in $X^{k}$ of a representative $k$-cell $\alpha$ must be invariant under the stabilizer in $\operatorname{Sym}(3) \times \operatorname{Alt}(4)$ of $\alpha$. Moreover, because of the assumption (ii), the simplicial subdivision of the boundary of $\alpha$ in $X^{k}$ must be as given by $X^{k-1}$. Finally, the assumption (iv) forbids the occurrence of certain edges in $\hat{\alpha}$.

The Subdivision $X^{2}$ of $W^{2}$ : We need to subdivide the seven squares in Table 1. Each subdivision is specified by the choice of a diagonal. As mentioned above, we subdivide $S=x_{1} \times x_{1} x_{2} \times x_{3} x_{4}$ by choosing the diagonal $x_{123} x_{114}$. The diagonals of other squares are already forced by the argument in the proof of Lemma 3.1.

Subdivision $X^{3}$ of $W^{3}$ : Eight of the ten representative 3-cells of $W$ (from Table 1) are triangulated straightaway by an application of Lemma 2.2. In each case, it suffices to specify the points $x$ from which the cone over its antistar is to be taken. (Remember that the boundary of each of these cells is already triangulated by $X^{2}$.) These we choose as indicated in Table 2.

Table 2 Subdivisions of some 3-cells

\begin{tabular}{llll}
\hline Representative cell $\alpha$ & Choice of cone vertex $x$ & Representative cell $\alpha$ & Choice of cone vertex $x$ \\
\hline$x_{h} \times x_{1} x_{2} \times x_{1} x_{2} x_{3}$ & $x_{h 11}$ & $x_{h} \times x_{1} x_{4} \times x_{1} x_{2} x_{3}$ & $x_{h 11}$ \\
$x_{1} x_{2} \times x_{1} x_{2} \times x_{1} x_{2}$ & $x_{111}$ & $x_{1} x_{2} \times x_{1} x_{2} \times x_{1} x_{3}$ & $x_{111}$ \\
$x_{1} x_{2} \times x_{1} x_{2} \times x_{1} x_{4}$ & $x_{111}$ & $x_{1} x_{2} \times x_{1} x_{2} \times x_{3} x_{4}$ & $x_{114}$ \\
$x_{1} x_{2} \times x_{1} x_{3} \times x_{1} x_{4}$ & $x_{111}$ & $x_{1} x_{2} \times x_{2} x_{4} \times x_{3} x_{4}$ & $x_{144}$ \\
\hline
\end{tabular}


Table 3 Subdivisions of some 4-cells

\begin{tabular}{llll}
\hline Representative cell $\alpha$ & Choice of cone vertex $x$ & Representative cell $\alpha$ & Choice of cone vertex $x$ \\
\hline$x_{1} \times x_{1} x_{2} x_{3} \times x_{1} x_{2} x_{3}$ & $x_{111}$ & $x_{1} \times x_{1} x_{2} x_{3} \times x_{1} x_{2} x_{4}$ & $x_{111}$ \\
$x_{3} \times x_{1} x_{2} x_{3} \times x_{1} x_{2} x_{4}$ & $x_{311}$ & $x_{4} \times x_{1} x_{2} x_{3} \times x_{1} x_{2} x_{3}$ & $x_{411}$ \\
$x_{1} x_{2} \times x_{1} x_{2} \times x_{1} x_{2} x_{3}$ & $x_{111}$ & $x_{1} x_{2} \times x_{1} x_{2} \times x_{1} x_{3} x_{4}$ & $x_{111}$ \\
$x_{1} x_{2} \times x_{1} x_{3} \times x_{1} x_{2} x_{3}$ & $x_{111}$ & $x_{1} x_{2} \times x_{1} x_{3} \times x_{1} x_{3} x_{4}$ & $x_{111}$ \\
$x_{1} x_{2} \times x_{1} x_{4} \times x_{1} x_{3} x_{4}$ & $x_{111}$ & $x_{1} x_{2} \times x_{3} x_{4} \times x_{1} x_{2} x_{3}$ & $x_{111}$ \\
\hline
\end{tabular}

It is clear from the proof of Lemma 3.1 that $C$ cannot be triangulated without adding a new vertex, and by Lemma 3.2 (since $D$ is divided without adding any new vertex) that $E$ cannot be triangulated without a new vertex. Driven by the requirement of goodness, we insert the new 3-simplices $x_{132} x_{112} x_{133} x_{232}$ and $x_{213} x_{233} x_{212} x_{113}$ in $C$ (respectively, $x_{123} x_{133} x_{124} x_{223}$ and $x_{234} x_{224} x_{233} x_{134}$ in $E$ ). Notice that when these two simplices are sliced off from $C$ (respectively, from $E$ ), the resulting polytope has boundary $S_{2}^{0}\left(x_{112}, x_{233}\right) * S_{2}^{0}\left(x_{133}, x_{212}\right) * S_{2}^{0}\left(x_{232}, x_{113}\right)$ (respectively, $\left.S_{2}^{0}\left(x_{133}, x_{224}\right) * S_{2}^{0}\left(x_{124}, x_{233}\right) * S_{2}^{0}\left(x_{223}, x_{134}\right)\right)$. To complete the triangulation of $C$ (respectively, $E$ ), we form the cone over this new boundary from the new vertex $v_{321}$ (respectively, $u_{11}$ ).

Subdivision $X^{4}$ of $W^{4}$ : Ten of the eleven representative 4-cells from Table 1 may be triangulated using Lemma 2.2. The choice of the cone vertex is given in Table 3.

This method fails for the 4-cell $G=x_{1} x_{2} \times x_{1} x_{3} \times x_{2} x_{3} x_{4}$. (If we do the cone construction from any vertex then its boundary does not agree with $X^{3}[G]$.) We found the following ad hoc triangulation $\hat{G}$ of $G$ which fulfils all the requirements. The triangulation $\hat{G}$ contains the following 24 4-simplices:

$\begin{array}{lll}u_{64} v_{321} x_{112} x_{113} x_{133}, & u_{64} v_{321} x_{112} x_{113} x_{212}, & u_{64} v_{321} x_{112} x_{232} x_{133}, \\ u_{64} v_{321} x_{112} x_{232} x_{212}, & u_{64} v_{321} x_{233} x_{113} x_{133}, & u_{64} v_{321} x_{233} x_{113} x_{212}, \\ u_{64} v_{321} x_{233} x_{232} x_{133}, & u_{64} v_{321} x_{233} x_{232} x_{212}, & u_{64} x_{113} x_{114} x_{133} x_{233}, \\ u_{64} x_{114} x_{133} x_{134} x_{233}, & u_{64} x_{114} x_{134} x_{233} x_{234}, & u_{64} x_{112} x_{113} x_{114} x_{212}, \\ u_{64} x_{133} x_{134} x_{232} x_{233}, & u_{64} x_{134} x_{232} x_{233} x_{234}, & u_{64} x_{112} x_{113} x_{114} x_{133}, \\ u_{64} x_{112} x_{114} x_{133} x_{134}, & u_{64} x_{212} x_{232} x_{233} x_{234}, & u_{64} x_{112} x_{133} x_{134} x_{232}, \\ u_{64} x_{113} x_{114} x_{212} x_{233}, & u_{64} x_{114} x_{212} x_{233} x_{234}, & x_{132} x_{112} x_{133} x_{134} x_{232}, \\ x_{113} x_{114} x_{212} x_{213} x_{233}, & x_{114} x_{212} x_{213} x_{214} x_{233}, & x_{114} x_{212} x_{214} x_{233} x_{234} .\end{array}$

Subdivision $X^{5}$ of $W^{5}$ : Five of the six representative 5-cells from Table 1 may be triangulated using Lemma 2.2. The chosen cone vertex of each of $x_{1} x_{3} \times x_{1} x_{2} x_{3} \times$ $x_{2} x_{3} x_{4}, x_{1} x_{3} \times x_{1} x_{3} x_{4} \times x_{2} x_{3} x_{4}, x_{1} x_{3} \times x_{2} x_{3} x_{4} \times x_{2} x_{3} x_{4}, x_{3} x_{4} \times x_{1} x_{3} x_{4} \times x_{2} x_{3} x_{4}$, $x_{3} x_{4} \times x_{2} x_{3} x_{4} \times x_{2} x_{3} x_{4}$ is $x_{333}$. This method fails for the 5-cell $H=x_{1} x_{2} \times x_{1} x_{3} x_{4} \times$ 
$x_{2} x_{3} x_{4}$. Consider the 5-cell $K=x_{1} x_{2} x_{4} \times x_{2} x_{3} \times x_{1} x_{3} x_{4}$ in the $\operatorname{Sym}(3) \times \operatorname{Alt}(4)$-orbit of $H$. We found that

$$
\begin{aligned}
\hat{K}:=\left\{\alpha: x_{111} \alpha \text { is one of the first } 75\right. \text { facets in the description of } \\
\\
\left.\quad\left(S^{2} \times S^{2} \times S^{2}\right)_{124} \text { in Sect. 4.1 }\right\}
\end{aligned}
$$

is a triangulation of $K$ which fulfils all the requirements.

Subdivision $X^{6}$ of $W^{6}$ : All the three representative 6-cells from Table 1 may be triangulated using Lemma 2.2. In all the cases, we may choose $x_{111}$ as the cone vertex. From the subdivision of $x_{1} x_{2} x_{3} \times x_{1} x_{2} x_{3} \times x_{1} x_{2} x_{3}$ we get 1146 -simplices. These give $4 \times 114$ facets of $X^{6}$ which are the last nine orbits in the description of $\left(S^{2} \times S^{2} \times S^{2}\right)_{124}$ in Sect. 4.1. From the subdivision of $x_{1} x_{2} x_{3} \times x_{1} x_{2} x_{3} \times x_{1} x_{3} x_{4}$ we get 1306 -simplices. These give $36 \times 130$ facets of $X^{6}$ which are in 76 th to 140th orbits in the description of $\left(S^{2} \times S^{2} \times S^{2}\right)_{124}$. From the subdivision of $x_{1} x_{2} x_{3} \times x_{1} x_{2} x_{4} \times x_{1} x_{3} x_{4}$ we get 2256 -simplices. These give $24 \times 225$ facets of $X^{6}$ which are in the first 75 orbits in the description of $\left(S^{2} \times S^{2} \times S^{2}\right)_{124}$.

Now, it is not hard to verify that the resulting simplicial subdivision $X=X^{6}$ of $W$ (with a built-in isometry group $\operatorname{Sym}(3) \times \operatorname{Alt}(4)$ ) does have a good $\operatorname{Sym}(3)$-action. Hence Lemma 2.3 and Corollary 2.7 complete the proof.

Remark 3.4 One may proceed to triangulate the higher-dimensional skeletons of the face complex of the polytope $\left(\Delta^{3}\right)^{3}$ in a similar fashion. Indeed, each higherdimensional cell of this polytope contains a vertex of the form $x_{i i i}$ and one may form a cone over the boundary from such a vertex. This leads to a $\operatorname{Sym}(3) \times \operatorname{Alt}(4)$ invariant simplicial subdivision of $\left(\Delta^{3}\right)^{3}$ within which $\left(S^{2}\right)_{124}^{3}$ sits as a subcomplex.

\section{Triangulation of the Quotient Map $q: S^{2} \times S^{2} \times S^{2} \rightarrow \mathbb{C} P^{3}$}

\subsection{Description of $\left(S^{2} \times S^{2} \times S^{2}\right) 124$}

Its vertex-set is $V_{124}=\left\{x_{i j k}: 1 \leq i, j, k \leq 4\right\} \cup\left\{v_{p q r}: 1 \leq p, q, r \leq 4, p, q, r\right.$ are distinct $\} \cup\left\{u_{i j}: 1 \leq i, j \leq 6\right\}$. The group $\operatorname{Sym}(3) \times \operatorname{Alt}(4)$ acts as an automorphism group, where the actions of $\operatorname{Sym}(3)$ and Alt(4) on the vertices are as follows. On the vertices other than $u_{i j}$, $\operatorname{Sym}(3)$ acts by permuting the positions of the three subscripts while Alt(4) acts by permuting the values of these subscripts (which are elements of $\{1,2,3,4\})$. The action on the vertices $u_{i j}$ of the generators $\alpha=(1,2,3)$, $\beta=(1,2,4)$ of $\operatorname{Alt}(4)$ and $\gamma=(1,2,3), \delta=(1,2)$ of $\operatorname{Sym}(3)$ is as follows:

$$
\begin{aligned}
& \alpha=\prod_{i=1}^{6}\left(u_{i 1}, u_{i 2}, u_{i 4}\right)\left(u_{i 6}, u_{i 5}, u_{i+3,3}\right), \quad \beta=\prod_{i=1}^{6}\left(u_{i 1}, u_{i 3}, u_{i+3,2}\right)\left(u_{i 4}, u_{i 5}, u_{i+3,6}\right), \\
& \gamma=\prod_{i=1}^{6}\left(u_{1 i}, u_{2 i}, u_{3 i}\right)\left(u_{4 i}, u_{5 i}, u_{6 i}\right), \quad \delta=\prod_{i=1}^{6}\left(u_{1 i}, u_{6 i}\right)\left(u_{2 i}, u_{5 i}\right)\left(u_{3 i}, u_{4 i}\right) .
\end{aligned}
$$


(Here, summation in the subscripts is modulo 6.) Modulo this group the facets (i.e., maximal faces) are the following:

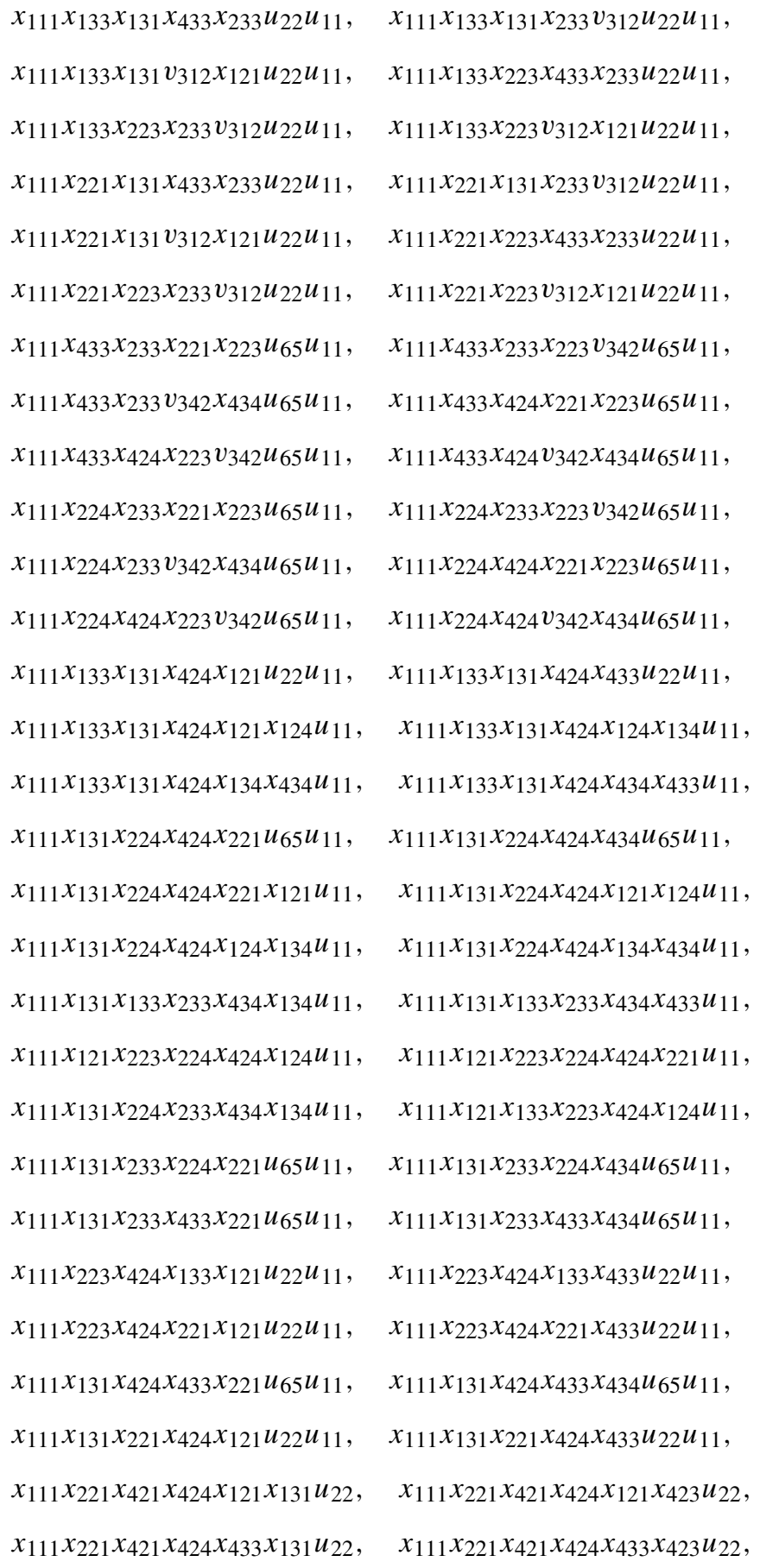


$x_{111} x_{223} x_{423} x_{424} x_{133} x_{121} u_{22}, \quad x_{111} x_{223} x_{423} x_{424} x_{133} x_{433} u_{22}$, $x_{111} x_{223} x_{423} x_{424} x_{221} x_{121} u_{22}, \quad x_{111} x_{223} x_{423} x_{424} x_{221} x_{433} u_{22}$, $x_{111} x_{131} x_{431} x_{433} x_{221} x_{231} u_{65}, \quad x_{111} x_{131} x_{431} x_{433} x_{221} x_{424} u_{65}$, $x_{111} x_{131} x_{431} x_{433} x_{434} x_{231} u_{65}, \quad x_{111} x_{131} x_{431} x_{433} x_{434} x_{424} u_{65}$, $x_{111} x_{131} x_{231} x_{233} x_{224} x_{221} u_{65}, \quad x_{111} x_{131} x_{231} x_{233} x_{224} x_{434} u_{65}$, $x_{111} x_{131} x_{231} x_{233} x_{433} x_{221} u_{65}, \quad x_{111} x_{131} x_{231} x_{233} x_{433} x_{434} u_{65}$, $x_{111} x_{131} x_{221} x_{421} x_{424} x_{431} x_{433}, \quad x_{111} x_{131} x_{224} x_{233} x_{234} x_{434} x_{134}$, $x_{111} x_{131} x_{224} x_{233} x_{234} x_{434} x_{231}, \quad x_{111} x_{121} x_{123} x_{133} x_{223} x_{424} x_{124}$, $x_{111} x_{121} x_{123} x_{133} x_{223} x_{424} x_{423}, \quad x_{111} x_{333} x_{114} x_{224} x_{334} x_{124} x_{134}$, $x_{111} x_{333} x_{114} x_{224} x_{334} x_{134} x_{234}, \quad x_{111} x_{333} x_{114} x_{224} x_{334} x_{234} x_{214}$, $x_{111} x_{333} x_{114} x_{123} x_{124} x_{133} x_{223}, \quad x_{111} x_{333} x_{114} x_{134} x_{224} x_{233} x_{234}$, $x_{111} x_{333} x_{114} x_{133} x_{124} x_{134} u_{11}, \quad x_{111} x_{333} x_{114} x_{133} x_{124} x_{223} u_{11}$, $x_{111} x_{333} x_{114} x_{133} x_{233} x_{134} u_{11}, \quad x_{111} x_{333} x_{114} x_{133} x_{233} x_{223} u_{11}$, $x_{111} x_{333} x_{114} x_{224} x_{124} x_{134} u_{11}, \quad x_{111} x_{333} x_{114} x_{224} x_{124} x_{223} u_{11}$, $x_{111} x_{333} x_{114} x_{224} x_{233} x_{134} u_{11}, \quad x_{111} x_{333} x_{114} x_{224} x_{233} x_{223} u_{11}$, $x_{111} x_{333} x_{113} x_{114} x_{223} x_{123} x_{133}, \quad x_{111} x_{333} x_{113} x_{114} x_{223} x_{133} x_{233}$, $x_{111} x_{333} x_{113} x_{114} x_{223} x_{233} x_{213}, \quad x_{111} x_{333} x_{114} x_{214} x_{233} x_{213} x_{223}$, $x_{111} x_{333} x_{114} x_{214} x_{233} x_{223} x_{224}, \quad x_{111} x_{333} x_{114} x_{214} x_{233} x_{224} x_{234}$, $x_{111} x_{333} x_{211} x_{233} x_{221} x_{223} x_{224}, \quad x_{111} x_{333} x_{211} x_{233} x_{214} x_{223} x_{224}$, $x_{111} x_{333} x_{211} x_{233} x_{213} x_{214} x_{223}, \quad x_{111} x_{333} x_{211} x_{233} x_{214} x_{224} x_{234}$, $x_{111} x_{333} x_{211} x_{233} x_{224} x_{231} x_{234}, \quad x_{111} x_{333} x_{211} x_{233} x_{221} x_{224} x_{231}$, $x_{111} x_{333} x_{223} x_{213} x_{211} x_{214} x_{313}, \quad x_{111} x_{333} x_{221} x_{224} x_{323} x_{321} x_{311}$, $x_{111} x_{333} x_{224} x_{323} x_{321} x_{324} x_{311}, \quad x_{111} x_{333} x_{224} x_{323} x_{324} x_{311} x_{314}$, $x_{111} x_{333} x_{223} x_{221} x_{224} x_{323} u_{61}, \quad x_{111} x_{333} x_{223} x_{221} x_{224} x_{211} u_{61}$, $x_{111} x_{333} x_{223} x_{224} x_{211} x_{214} u_{61}, \quad x_{111} x_{333} x_{223} x_{211} x_{214} x_{313} u_{61}$, $x_{111} x_{333} x_{221} x_{224} x_{323} x_{311} u_{61}, \quad x_{111} x_{333} x_{221} x_{224} x_{211} x_{311} u_{61}$, $x_{111} x_{333} x_{224} x_{211} x_{214} x_{311} u_{61}, \quad x_{111} x_{333} x_{224} x_{214} x_{311} x_{314} u_{61}$, $x_{111} x_{333} x_{224} x_{323} x_{311} x_{314} u_{61}, \quad x_{111} x_{333} x_{211} x_{214} x_{313} x_{311} u_{61}$, $x_{111} x_{333} x_{214} x_{313} x_{311} x_{314} u_{61}, \quad x_{111} x_{333} x_{323} x_{313} x_{311} x_{314} u_{61}$, $x_{111} x_{333} x_{223} x_{221} x_{211} v_{132} u_{61}, \quad x_{111} x_{333} x_{223} x_{221} x_{323} v_{132} u_{61}$, $x_{111} x_{333} x_{223} x_{313} x_{211} v_{132} u_{61}, \quad x_{111} x_{333} x_{223} x_{313} x_{323} v_{132} u_{61}$, 


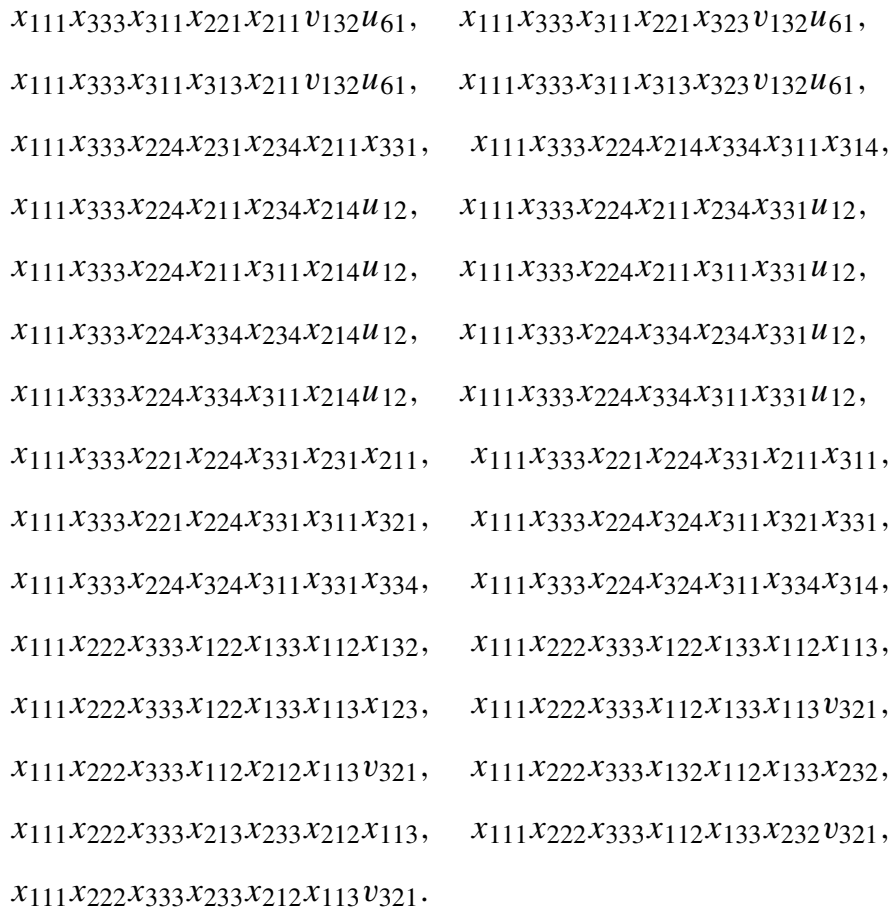

The full list of facets of $\left(S^{2} \times S^{2} \times S^{2}\right) 124$ may be obtained from these 149 basic facets by applying the group $\operatorname{Sym}(3) \times \operatorname{Alt}(4)$. Under this group, the first 145 basic facets form orbits of length 72 each, while each of the last 4 forms an orbit of length 24 , yielding a total of $145 \times 72+4 \times 24=10536$ facets. The face vector of $\left(S^{2} \times S^{2} \times S^{2}\right)_{124}$ is $(124,1908,11740,34140,50532,36876,10536)$. The group $\operatorname{Sym}(3) \times \operatorname{Alt}(4)$ appears to be its full group of automorphisms. In Theorem 1.1 we have shown that this simplicial complex triangulates $S^{2} \times S^{2} \times S^{2}$.

\subsection{Description of $\mathbb{C} P_{30}^{3}$}

Let $V_{124}$ and $\left(S^{2} \times S^{2} \times S^{2}\right)_{124}$ be as above. Let

$$
q: V_{124} \longrightarrow V_{30}:=V_{124} / \operatorname{Sym}(3)
$$

be the quotient map. Explicitly,

$$
\begin{aligned}
& V_{30}=\left\{x_{i j}: 1 \leq i, j \leq 4\right\} \sqcup\left\{y_{i}: 1 \leq i \leq 4\right\} \sqcup\left\{v_{i}: 1 \leq i \leq 4\right\} \sqcup\left\{u_{j}: 1 \leq j \leq 6\right\}, \\
& \text { where } x_{i j}=q\left(x_{i i j}\right)=q\left(x_{i j i}\right)=q\left(x_{j i i}\right), \quad 1 \leq i, j \leq 4, \\
& \qquad y_{i}=q\left(x_{j k l}\right), \quad i j k l \text { is a permutation of } 1234, \\
& v_{i}=q\left(v_{j k l}\right), \quad i j k l \text { is a permutation of } 1234, \\
& u_{j}=q\left(u_{i j}\right), \quad 1 \leq i, j \leq 6 .
\end{aligned}
$$

Thus $\mathbb{C} P_{30}^{3}$, the quotient of $\left(S^{2} \times S^{2} \times S^{2}\right)_{124}$ by $\operatorname{Sym}(3)$, is a 30 -vertex triangulation of $\mathbb{C} P^{3}$ with vertex-set $V_{30}$. Since the automorphism groups $\operatorname{Alt}(4)$ and $\operatorname{Sym}(3)$ of 
$\left(S^{2} \times S^{2} \times S^{2}\right)_{124}$ commute, its Alt(4)-action induces an $\operatorname{Alt}(4)$-action on $\mathbb{C} P_{30}^{3}$. Explicitly, if $\alpha, \beta$ are the generators of Alt(4) given by $\alpha=(1,2,3), \beta=(1,2,4)$, then $\alpha, \beta$ act on $V_{30}$ by:

$$
\begin{aligned}
\alpha= & \left(x_{11}, x_{22}, x_{33}\right)\left(x_{12}, x_{23}, x_{31}\right)\left(x_{13}, x_{21}, x_{32}\right)\left(x_{14}, x_{24}, x_{34}\right)\left(x_{41}, x_{42}, x_{43}\right) \\
& \times\left(y_{1}, y_{2}, y_{3}\right)\left(v_{1}, v_{2}, v_{3}\right)\left(u_{1}, u_{2}, u_{4}\right)\left(u_{3}, u_{6}, u_{5}\right), \\
\beta= & \left(x_{11}, x_{22}, x_{44}\right)\left(x_{12}, x_{24}, x_{41}\right)\left(x_{21}, x_{42}, x_{14}\right)\left(x_{13}, x_{23}, x_{43}\right)\left(x_{31}, x_{32}, x_{34}\right) \\
& \times\left(y_{1}, y_{2}, y_{4}\right)\left(v_{1}, v_{2}, v_{4}\right)\left(u_{1}, u_{3}, u_{2}\right)\left(u_{4}, u_{5}, u_{6}\right) .
\end{aligned}
$$

Modulo Alt $(4)=\langle\alpha, \beta\rangle$ the basic facets of $\mathbb{C} P_{30}^{3}$ are the images under $q$ of the 149 basic facets in Sect. 4.1:

$$
\begin{gathered}
x_{11} x_{31} x_{13} x_{34} x_{32} u_{2} u_{1}=q\left(x_{111} x_{133} x_{131} x_{433} x_{233} u_{22} u_{11}\right), \\
\vdots \\
x_{11} x_{22} x_{33} x_{32} x_{21} x_{13} v_{4}=q\left(x_{111} x_{222} x_{333} x_{233} x_{212} x_{113} v_{321}\right) .
\end{gathered}
$$

The full list of facets of $\mathbb{C} P_{30}^{3}$ may be obtained from these 149 basic facets by applying the group Alt(4). Under this group, the first 145 basic facets form orbits of length 12 each, while each of the last 4 forms an orbit of length 4 , yielding a total of $145 \times 12+4 \times 4=1756$ facets. The face vector of $\mathbb{C} P_{30}^{3}$ is $(30,362,2066,5810,8470,6146,1756)$. The group Alt(4) is the full group of automorphisms of this simplicial complex (verified by the simpcomp program of Effenberger and Spreer [7]).

\subsection{The Branch Locus of the Quotient Map $q:\left(S^{2} \times S^{2} \times S^{2}\right)_{124} \rightarrow \mathbb{C} P_{30}^{3}$}

Notice that the quotient map $S^{2} \times S^{2} \times S^{2} \rightarrow \mathbb{C} P^{3}$, which is generically six-to-one (given by Lemma 2.3), branches over the union of three copies of $S^{2} \times S^{2}$ any two of which intersect in the diagonal $S^{2}$. It is interesting to examine how this phenomenon is reflected in the simplicial covering map $q:\left(S^{2} \times S^{2} \times S^{2}\right)_{124} \rightarrow \mathbb{C} P_{30}^{3}$.

Let $V^{i}, i=1,2,3$, be the three 16 -vertex subsets of $V_{124}$ given by

$$
\begin{aligned}
& V^{1}=\left\{x_{i j k}: j=k, 1 \leq i, j, k \leq 4\right\}, \\
& V^{2}=\left\{x_{i j k}: i=k, 1 \leq i, j, k \leq 4\right\}, \\
& V^{3}=\left\{x_{i j k}: i=j, 1 \leq i, j, k \leq 4\right\} .
\end{aligned}
$$

We have $V^{j} \cap V^{k}=V^{0}:=\left\{x_{i i i}: 1 \leq i \leq 4\right\}$ for $1 \leq j \neq k \leq 3$. Let $X_{i}$ be the induced subcomplex of $\left(S^{2} \times S^{2} \times S^{2}\right)_{124}$ on $V^{i}, 0 \leq i \leq 3$. Then the formula (1) shows that $q$ is three-to-one on $V^{1} \cup V^{2} \cup V^{3} \backslash V^{0}$ and one-to-one on $V^{0}$. It is six-toone outside $V^{1} \cup V^{2} \cup V^{3}$. Thus the branch locus passes through the 40 vertices in $V^{1} \cup V^{2} \cup V^{3}$. The induced subcomplex $X_{i}$ on each $V^{i}(1 \leq i \leq 3)$ is a copy of $\left(S^{2} \times S^{2}\right)_{16}$, while the induced subcomplex $X_{0}$ on $V^{0}$ is $S_{4}^{2}$. Each $X_{i}, 1 \leq i \leq 3$, is mapped isomorphically onto a copy of $\left(S^{2} \times S^{2}\right)_{16}$ in $\mathbb{C} P_{30}^{3}$. (This is the $\left(S^{2} \times S^{2}\right)_{16}$ discussed in [3].) Thus the combinatorics is a perfect reflection of the topology in this construction. 


\section{An 18-Vertex Triangulation of $\mathbb{C} P^{3}$}

We applied the BISTELLAR program of Lutz [9] on $\mathbb{C} P_{30}^{3}$. The BISTELLAR flips were carried out on a PC with Intel Atom processor $(1.66 \mathrm{Ghz})$ for approximately 16 hours. The final output was an 18 -vertex simplicial complex $\mathbb{C} P_{18}^{3}$ which is bistellar equivalent to $\mathbb{C} P_{30}^{3}$ and hence triangulates $\mathbb{C} P^{3}$. Its face vector is $(18,153,783,2110,3021,2177,622)$. Its automorphism group is trivial (verified by the simpcomp program of Effenberger and Spreer [7]). For the complete list of the 622 facets of $\mathbb{C} P_{18}^{3}$, see [4].

Recall that Kühnel's $\mathbb{C} P_{9}^{2}$ on the vertices $b_{1}, \ldots, b_{9}$ has facets

$\begin{array}{lllll}b_{1} b_{2} b_{3} b_{7} b_{8}, & b_{1} b_{2} b_{3} b_{7} b_{9}, & b_{1} b_{2} b_{3} b_{8} b_{9}, & b_{1} b_{2} b_{4} b_{5} b_{6}, & b_{1} b_{2} b_{4} b_{5} b_{9}, \\ b_{1} b_{2} b_{4} b_{6} b_{7}, & b_{1} b_{2} b_{4} b_{7} b_{9}, & b_{1} b_{2} b_{5} b_{6} b_{8}, & b_{1} b_{2} b_{5} b_{8} b_{9}, & b_{1} b_{2} b_{6} b_{7} b_{8}, \\ b_{1} b_{3} b_{4} b_{5} b_{6}, & b_{1} b_{3} b_{4} b_{5} b_{7}, & b_{1} b_{3} b_{4} b_{6} b_{8}, & b_{1} b_{3} b_{4} b_{7} b_{8}, & b_{1} b_{3} b_{5} b_{6} b_{9}, \\ b_{1} b_{3} b_{5} b_{7} b_{9}, & b_{1} b_{3} b_{6} b_{8} b_{9}, & b_{1} b_{4} b_{5} b_{7} b_{9}, & b_{1} b_{4} b_{6} b_{7} b_{8}, & b_{1} b_{5} b_{6} b_{8} b_{9}, \\ b_{2} b_{3} b_{4} b_{5} b_{6}, & b_{2} b_{3} b_{4} b_{5} b_{8}, & b_{2} b_{3} b_{4} b_{6} b_{9}, & b_{2} b_{3} b_{4} b_{8} b_{9}, & b_{2} b_{3} b_{5} b_{6} b_{7}, \\ b_{2} b_{3} b_{5} b_{7} b_{8}, & b_{2} b_{3} b_{6} b_{7} b_{9}, & b_{2} b_{4} b_{5} b_{8} b_{9}, & b_{2} b_{4} b_{6} b_{7} b_{9}, & b_{2} b_{5} b_{6} b_{7} b_{8}, \\ b_{3} b_{4} b_{5} b_{7} b_{8}, & b_{3} b_{4} b_{6} b_{8} b_{9}, & b_{3} b_{5} b_{6} b_{7} b_{9}, & b_{4} b_{5} b_{7} b_{8} b_{9}, & b_{4} b_{6} b_{7} b_{8} b_{9}, \\ b_{5} b_{6} b_{7} b_{8} b_{9} . & & & & \end{array}$

The 18 -vertex $\mathbb{C} P_{18}^{3}$ obtained by us has vertices $a_{1}, \ldots, a_{9}, b_{1}, \ldots, b_{9}$ and its induced subcomplex on the $b_{i}$ 's is the $\mathbb{C} P_{9}^{2}$ above. The simplicial complement of this $\mathbb{C} P_{9}^{2}$ in $\mathbb{C} P_{18}^{3}$ is the 6-ball with vertices $a_{1}, \ldots, a_{9}$ and facets

$\begin{array}{llll}a_{1} a_{2} a_{3} a_{4} a_{5} a_{7} a_{8}, & a_{1} a_{2} a_{3} a_{4} a_{5} a_{7} a_{9}, & a_{1} a_{2} a_{3} a_{4} a_{5} a_{8} a_{9}, & a_{1} a_{2} a_{3} a_{4} a_{6} a_{7} a_{8}, \\ a_{1} a_{2} a_{3} a_{4} a_{6} a_{7} a_{9}, & a_{1} a_{2} a_{3} a_{4} a_{6} a_{8} a_{9}, & a_{1} a_{2} a_{3} a_{5} a_{6} a_{7} a_{9} .\end{array}$

Acknowledgements The authors thank the anonymous referee for some useful comments which led to substantial improvements in the presentation of this paper. The authors are thankful to Nitin Singh for his help in running the BISTELLAR and simpcomp programs. The second author was partially supported by UGC-SAP/DSA-IV and a grant from UGC Centre for Advanced Study.

\section{References}

1. Arnoux, P., Marin, A.: The Kühnel triangulation of complex projective plane from the view-point of complex crystallography (part II). Mem. Fac. Sci., Kyushu Univ., Ser. A, Math. 45, 167-244 (1991)

2. Bagchi, B., Datta, B.: On Kühnel's 9-vertex complex projective plane. Geom. Dedic. 50, 1-13 (1994)

3. Bagchi, B., Datta, B.: From the icosahedron to natural triangulations of $\mathbb{C} P^{2}$ and $S^{2} \times S^{2}$. Discrete Comput. Geom. 46, 542-560 (2011)

4. Bagchi, B., Datta, B.: A triangulation of $\mathbb{C} P^{3}$ as symmetric cube of $S^{2}$ (2010). arXiv:1012.3235v1, 29 pp.

5. Banchoff, T.F., Kühnel, R.: Equilibrium triangulations of the complex projective plane. Geom. Dedic. 44, 413-433 (1992)

6. Datta, B.: Minimal triangulations of manifolds. IISc J. 87, 429-449 (2007)

7. Effenberger, F., Spreer, J.: simpcomp-A GAP toolkit for simplicial complexes, version 1.5.4 (2011). http://www.igt.uni-stuttgart.de/LstDiffgeo/simpcomp. http://code.google.com/p/simpcomp/

8. Kühnel, W., Banchoff, T.F.: The 9-vertex complex projective plane. Math. Intell. 5(3), 11-22 (1983) 
9. Lutz, F.H.: BISTELLAR, version Nov/2003. http://www.math.TU-Berlin.de/diskregeom/stellar/ BISTELLAR

10. Sergeraert, F.: Triangulations of complex projective spaces. In: Lambán, L., et al. (eds.) Contributiones Científicas en Honor de Mirian Andrés Gómez. Serv. Publ., pp. 507-519. University of La Rioja, Logroño (2010)

11. Ziegler, G.M.: Lectures on Polytopes. Springer, New York (1995) 